\title{
Synthesis, Characterization, Properties, and Applications of Nanosized Ferroelectric, Ferromagnetic, or Multiferroic Materials
}

\author{
Debasis Dhak, ${ }^{1}$ Seungbum Hong, ${ }^{2}$ Soma Das, ${ }^{3}$ and Prasanta Dhak ${ }^{4}$ \\ ${ }^{1}$ Department of Chemistry, Sidho-Kanho-Birsha University, Purulia, West Bengal 723101, India \\ ${ }^{2}$ Materials Science Division, Argonne National Laboratory, Lemont, IL 60439, USA \\ ${ }^{3}$ Electronics \& Communication Engineering, Institute of Technology, Guru Ghasidas Vishwavidyalaya, Bilaspur 495009, India \\ ${ }^{4}$ Department of Materials Science and Engineering, Seoul National University, Seoul 151-744, Republic of Korea \\ Correspondence should be addressed to Debasis Dhak; debasisdhak@yahoo.co.in \\ Received 28 December 2014; Accepted 28 December 2014 \\ Copyright (c) 2015 Debasis Dhak et al. This is an open access article distributed under the Creative Commons Attribution License, \\ which permits unrestricted use, distribution, and reproduction in any medium, provided the original work is properly cited.
}

Recently, there has been an enormous increase in research activity in the field of ferroelectrics and ferromagnetics especially in multiferroic materials which possess both ferroelectric and ferromagnetic properties simultaneously. However, the ferroelectric, ferromagnetic, and multiferroic properties should be further improved from the utilitarian and commercial viewpoints.

Nanostructural materials are central to the evolution of future electronics and information technologies. Ferroelectrics and ferromagnetics have already been established as a dominant branch in electronics sector because of their diverse applications. The ongoing dimensional downscaling of materials to allow packing of increased numbers of components into integrated circuits provides the momentum for evolution of nanostructural devices. Nanoscaling of the above materials can result in a modification of their functionality. Furthermore, nanoscaling can be used to form high density arrays of nanodomain nanostructures, which is desirable for miniaturization of devices.

In the paper "Characterization of Multiferroic Domain Structures in Multiferroic Oxides," L. Liang et al. have reviewed the recent progress in the characterizations of multiferroic domain structures in multiferroic oxides. Due to the existence of two or three primary ferroic order parameters simultaneously in the same phase multiferroics and intricately coupling each other, new amazing multiferroic properties and/or phenomena are realized by combinations of distinct nanometer-scale charge-ordered domains, cloverleaf domain structures, and topological defects such as multiferroic vortex-antivortex pairs. Recent development of spherical aberration correction is revolutionizing the performance of HRTEM/STEM instruments, which allows one to achieve a spatial resolution better than $0.08 \mathrm{~nm}$ and an energy resolution better than $100 \mathrm{MeV}$. Such breakthrough would bring us to see and thoroughly explore the multiferroic domain structures at subangstrom scale.

In the paper "Enhanced Magnetization of Sol-Gel Synthesized Pb-Doped Strontium Hexaferrites Nanocrystallites at Low Temperature," S. M. Ramay et al. have shown the influence of $\mathrm{Pb}$ doping on the structural and low temperature magnetic behavior of $\mathrm{SrPb}_{x} \mathrm{Fe}_{12-x} \mathrm{O}_{19}$. Saturation magnetization was found to decrease while coercivity was increased with the increase of $\mathrm{Pb}$ contents in the series at $300 \mathrm{~K}$. When temperature was reduced to $200 \mathrm{~K}$, the saturation magnetization of the relevant samples was increased, credited to the preferred individual spin alignments at low temperature. Remanence was also decreased but the corresponding effect was less prominent as the $\mathrm{M}_{\mathrm{r}} / \mathrm{M}_{\mathrm{s}}$ and consequently the energy product were found to increase, corroborating the use of these ferrite compositions where hard magnetic characteristics are required.

In the paper "Fabrication, Characterization, Properties, and Applications of Low-Dimensional $\mathrm{BiFeO}_{3}$ Nanostructures," $\mathrm{H}$. Wu et al. also have reviewed the recent research progress of low-dimensional BFO nanostructures, including their fabrication, property, structural characterization, and 
applications. Perovskite-type BFO as one of the few known single-phase multiferroics possesses ferroelectricity and antiferromagnetism at room temperature. Its low-dimensional nanostructures are much attractive in the applications of multistate storage, magnetoelectric sensor, and spintronic devices.

In the paper "Ferroelectric and Dielectric Properties of $\mathrm{ZnFe}_{2} \mathrm{O}_{4}-\mathrm{Pb}(\mathrm{ZrTi}) \mathrm{O}_{3}$ Multiferroic Nanocomposites," S. Sen et al. have synthesized $\mathrm{ZnFe}_{2} \mathrm{O}_{4}-\mathrm{Pb}(\mathrm{ZrTi}) \mathrm{O}_{3}$ by solgel technique and have characterized them by XRD, TEM, and SEM for structural and microstructural analysis. The dielectric constant along with the P-E hysteresis loops was also studied for the mentioned samples.

In the paper "Growth of $\mathrm{BiFeO}_{3}$ Microcylinders under a Hydrothermal Condition,” L. J. Di et al. demonstrated the hydrothermal synthesis of cylinder-like BFO microcylinders, size of which is dependent on hydrothermal reaction time. Photocatalytical experimental results demonstrate that the BFO microcylinders exhibit an appreciable photocatalytic activity toward the degradation of "acid orange 7 " under simulated sunlight irradiation. Magnetic hysteresis loop measurement reveals an antiferromagnetic behavior in the $\mathrm{BiFeO}_{3}$ microcylinders at room temperature.

As the sizes of the microelectronics enter into nanoscale, there are still some problems that need to be solved in fabrication, characterization, and application of $\mathrm{BiFeO}_{3}$ and other low-dimensional nanostructures. For instance, in BFO nanostructures there exists quantum size effect (ferroelectric and magnetoelectric), size effect, and surface/interface effect; all these effects must be considered together from experimental and theoretical researches, which are the fundamental to develop the new generation of revolutionary electronic nanodevices. Although the BFO has good ferroelectricity, its weak ferromagnetism is highly required to be enhanced, which could be achieved in low-dimensional nanostructures. Therefore, deeper understanding of the fundamentals of the low-dimensional nanostructures with the development of advanced technology and exploring the coexistence of ferroelectricity and ferromagnetism with strong coupling between them will be the future direction of multiferroic nanomaterials researches.

Another fascinating task is to combine the measurements of multiferroic physical properties (e.g., ferroelectric, magnetic, and magnetoelectric coupling properties) with the multiferroic domain analysis as focused on here in this special issue. This type of simultaneous measurements will be necessary for further understanding of the ferroelectric, magnetic, and/or magnetoelectric coupling between the small domains. An exciting new era for multiferroic domain characterizations in multiferroic oxides is on the horizon.

In summary, as reflected by its content this special issue provides a broad panorama for research in this specific field and will benefit the readers, especially those working in this field.

\section{Acknowledgments}

We would like to thank our reviewers for their time and comments and thank the authors for their contributions to this special issue. This special issue could not have been successful without their contribution and support. The work at Argonne (Seungbum Hong was responsible for paper writing) was supported by the US Department of Energy, Office of Science, Materials Sciences and Engineering Division.

Debasis Dhak Seungbum Hong Soma Das Prasanta Dhak 

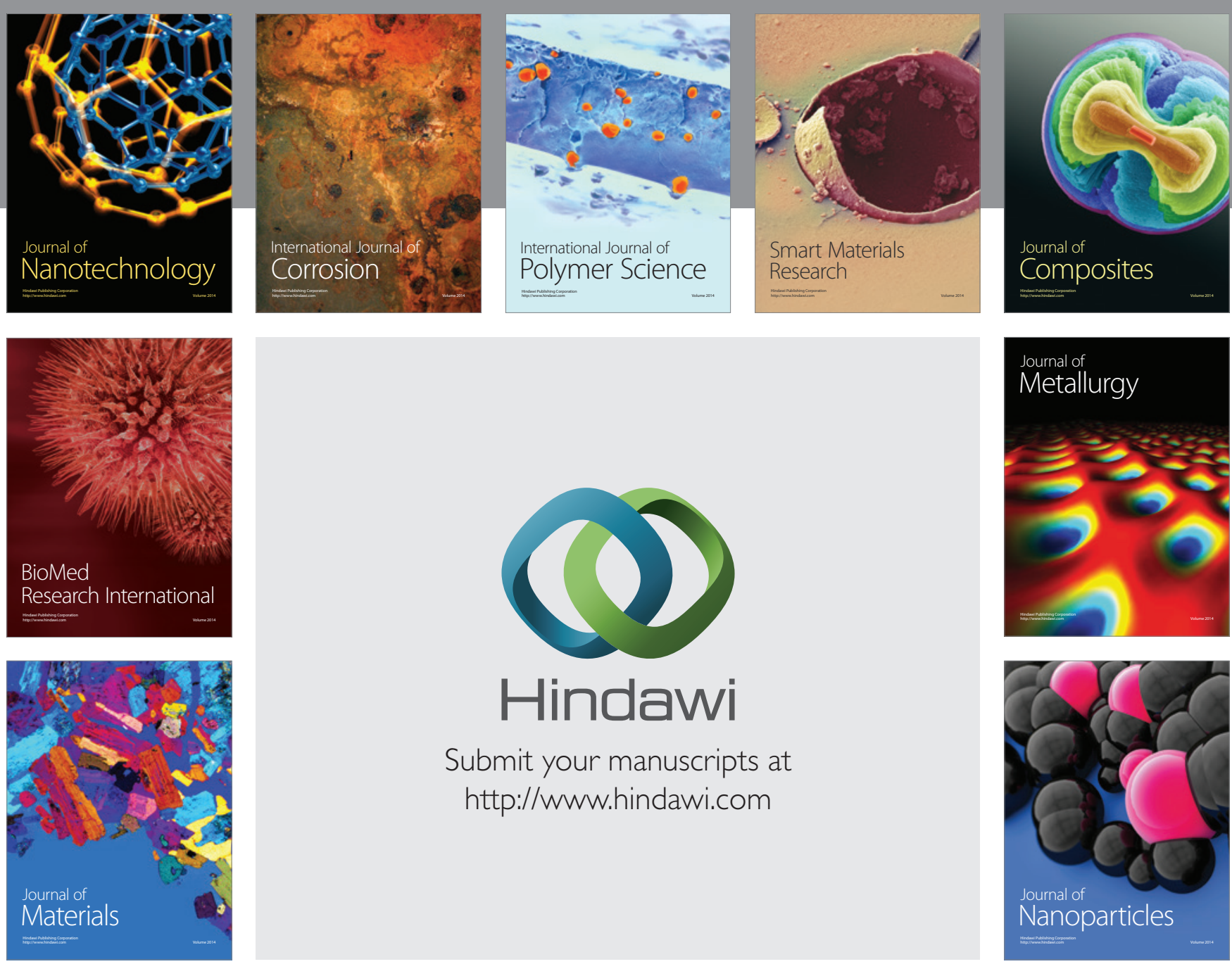

Submit your manuscripts at http://www.hindawi.com
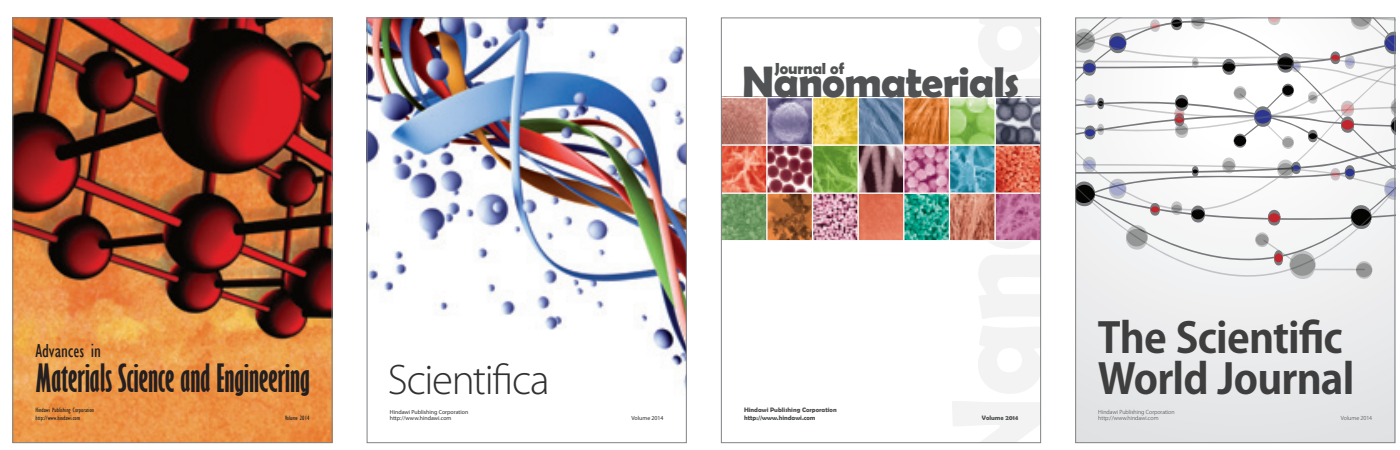

\section{The Scientific World Journal}
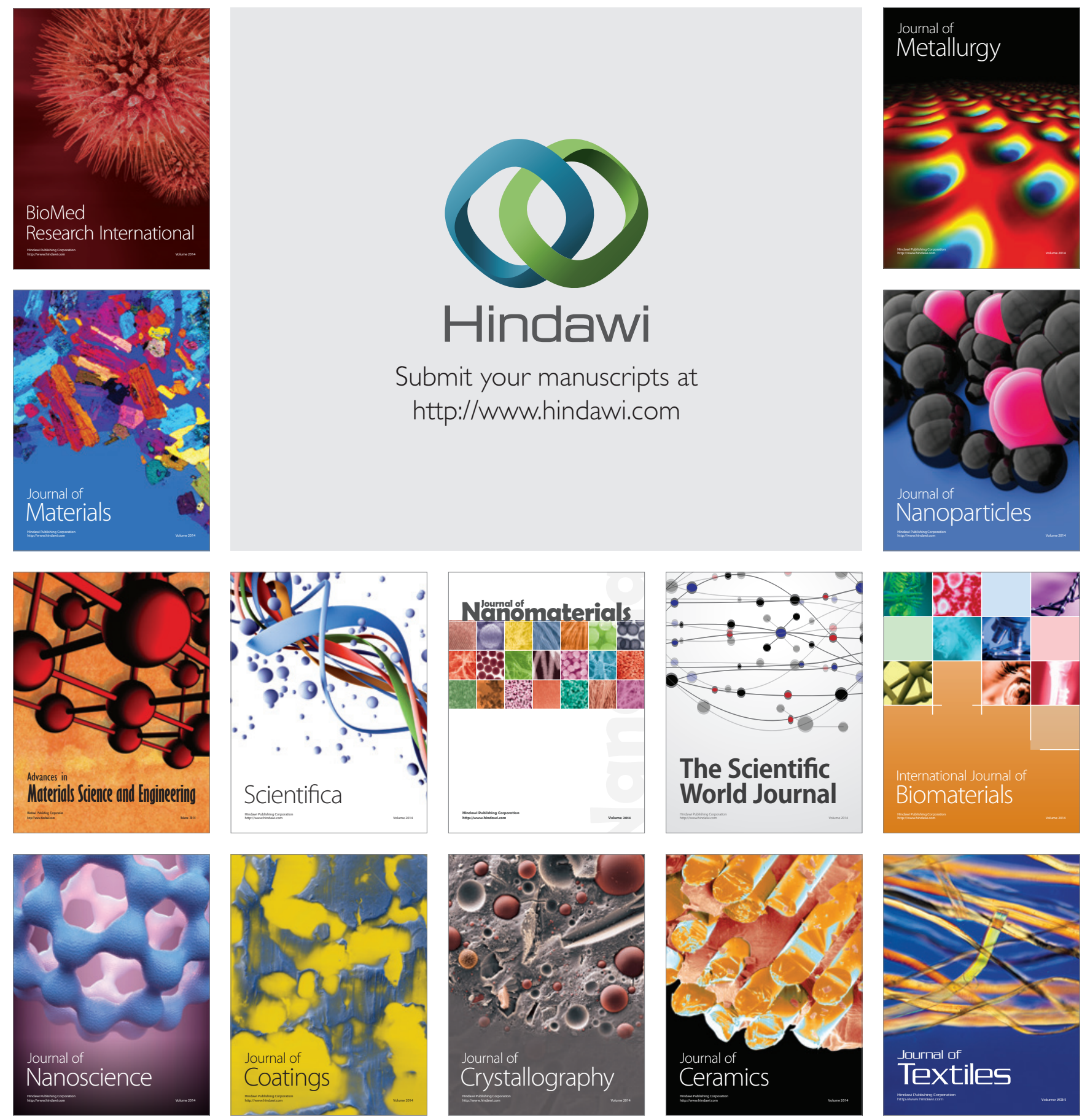